\title{
Analytic Modeling for Grant-Free Transmission in Cell-Free Massive MIMO: A Stochastic Geometry Approach
}

This paper was downloaded from TechRxiv (https://www.techrxiv.org).

\section{LICENSE}

CC BY 4.0

SUBMISSION DATE / POSTED DATE

03-09-2021 / 09-09-2021

\section{CITATION}

Ding, Jie; Homssi, Bassel Al; Choi, Jinho (2021): Analytic Modeling for Grant-Free Transmission in Cell-Free Massive MIMO: A Stochastic Geometry Approach. TechRxiv. Preprint. https://doi.org/10.36227/techrxiv.16566201.v1

DOI 


\title{
Analytic Modeling for Grant-Free Transmission in Cell-Free Massive MIMO: A Stochastic Geometry Approach
}

\author{
Jie Ding, Bassel Al Homssi, Member, IEEE, and Jinho Choi, Senior \\ Member, IEEE
}

\begin{abstract}
Cell-free (CF) massive multiple-input multiple-output (MIMO), as a promising network architecture for beyond the fifth generation $(5 \mathrm{G})$, has a great potential to support grant-free (GF) transmission for machine-type communication (MTC). To shed light on this subject, this work aims to model and evaluate the performance of GF transmission in CF massive MIMO under a realistic network deployment scenario, where the spatial locations of both access points (APs) and devices are assumed to be random in nature. In particular, by capitalizing on the distinctive CF network architecture and features, we design a new two-disk based geometric model for GF transmission, which facilitates analysis and understanding in CF massive MIMO. Based on the proposed two-disk model, we derive an approximated closed-form expression for the access success probability by leveraging on techniques from stochastic geometry, and investigate the impact of different key system parameters on the network performance. To highlight the performance superiority of CF massive MIMO, we further provide a comparative analysis by using an analogous single-disk model in an equivalent co-located massive MIMO network. Simulation results verify our analysis and demonstrate that $\mathrm{CF}$ massive MIMO is able to significantly outperform its co-located counterpart in terms of access success probability and provide robust performance against increased access density, which well suits to crowd scenarios.
\end{abstract}

\section{Index Terms}

Grant-free transmission; random access; stochastic geometry; modeling; cell-free massive MIMO; IoT

The authors are with the School of Information Technology, Deakin University, Geelong, VIC 3220, Australia. 


\section{INTRODUCTION}

The excitement about the fifth generation $(5 \mathrm{G})$ is ushering the possibility of providing allpurpose connectivities for a wide range of Internet of Things (IoT) services such as smart home, factory automation, smart farm, and intelligent transportation [1]. As a key enabler of IoT, machine-type communication (MTC) has drawn significant attention in the research community and industry in the last decade and will continue to play an important integral role in the provision of ubiquitous connectivity in the global scale for beyond 5G [2]. Different from conventional human-type communication, MTC is dominated by more uplink traffic driven by a large number of autonomous devices which usually have low duty cycles while transmitting short bursts of data [3]. As such, there is increasing interest in the development of various media access protocols based on random access for MTC.

Due to limited capabilities and energy, MTC devices are triggered to perform random access when they have new data packets to transmit. To accommodate MTC with small payloads, grant-free (GF) random access has been adopted in 5G new radio [4]. Compared to conventional grant-based random access, GF random access can reduce signaling overhead and access delay since devices do not need to perform grant acquisition handshaking with a base station (BS) and wait for a scheduling grant from the BS for data transmissions [5]. In particular, once devices become active in GF random access, each of the devices can directly transmit a randomly selected preamble from an orthogonal preamble pool followed by a data packet [6]. In particular, according to the 3rd generation partnership project (3GPP) standard [4], orthogonal Zadoff-Chu (ZC) sequences are used as preambles thanks to their good auto-correlation properties. Due to the nature of GF transmission in random access, a number of sporadically active devices may have to contend for the shared channel resources. As a result, the BS has to identify the devices and estimate their channel state information (CSI) by detecting the preambles before attempting to decode any data using coherent decoding. When a preamble collision does occur, i.e., multiple 
devices select the same preamble over the same channel resources, the estimated CSI becomes a noisy superposition of multiple channels of the collided devices, which can lead to unsuccessful data decoding [7]. Therefore, advanced technologies that can provide high spectral efficiency and address the preamble collision issue for the GF transmission are desired.

Massive multiple-input multiple-output (MIMO) technology [8] can be exploited to enable efficient GF transmissions in random access [6]. Conventionally, massive MIMO is also referred to as co-located massive MIMO, where a large number of antennas are co-located at a BS. Colocated massive MIMO is able to provide two prominent features such as channel hardening and favorable propagation so that several devices sharing the same channel resources can be served simultaneously [9], [10]. By taking advantage of these features, various designs and approaches in GF random access, in terms of protocol modification [11], preamble design [12] [13], and frame structure [14], have been developed to alleviate preamble collision and its impact on performance. To further resolve the preamble collision when it occurs, a successive interference cancellations (SIC) based scheme was proposed in [15]. Nevertheless, it is built on multipleslot transmissions, i.e., active devices need to transmit multiple preambles and same data over multiple slots to make SIC feasible. In fact, in the context of co-located massive MIMO, it is essentially difficult to resolve the preamble collision on the basis of a single random access slot. This is due to the fact that co-located massive MIMO network is a BS-centric network and all the signals of collided devices are eventually received by all the serving antennas at a centralized BS. Thus, the resulting contamination in channel estimation and coherent interference in data decoding cannot be resolved.

An alternative to allocating antennas in a co-located fashion is to deploy a distributed network with a large number of geographically distributed access points (APs) to jointly serve devices [16], [17]. Recently, cell-free (CF) massive MIMO, a new incarnation of distributed massive MIMO without the concepts of cell boundaries [18], has emerged as an alternative 
deployment to traditional co-located massive MIMO for beyond 5G networks [19]-[21]. In CF massive MIMO, macro diversity and signal spatial sparsity [22], [23] are the most two distinctive features differentiating it from co-located massive MIMO, which can be exploited to resolve the preamble collision issue in GF random access [24]. Unfortunately, so far there is still little research on this subject and lacks of tailored modeling built on the above distinctive features for the GF transmission. In fact, most existing related studies in CF massive MIMO only focus on preamble assignment optimization for a fixed number of devices prior to their uplink transmissions [18], [25]-[27], by relying on the condition that the full or partial CSI of devices is known in advance at the BS, which is however not the case in random access for sporadic MTC. In addition, in realistic network deployment of CF massive MIMO, the numbers of APs and sporadically active devices can vary and both their locations follow irregular and stochastic patterns. Thus, their randomness and uncertainty need to be captured to characterize the impact of preamble collision and analyze the performance of GF transmission in CF massive MIMO, which is however beyond the scope of the existing works.

Motivated by these literature gaps, this work proposes a new analytic model that depicts a realistic GF transmission in CF massive MIMO network architecture. Based on the proposed model and by leveraging on tools from stochastic geometry, a theoretical analysis incorporating the network irregularity in terms of geometric distribution is provided to evaluate and identify the realistic potentials of CF massive MIMO. In particular, the novelty and main contributions of this work are summarized as follows.

- We design a novel two-disk based geometric model for the GF transmission on the basis of macro diversity and signal spatial sparsity in CF massive MIMO, which facilitates analysis and insights into the added benefits of CF massive MIMO.

- Based on the two-disk model, we present a tractable performance analysis in CF massive MIMO by considering the stochastic distributions of randomly deployed APs and 
devices. We analytically derive an accurate approximated expression for the access success probability and provide insights into its performance behaviors with respect to key system parameters.

- We further derive a closed-form expression for an asymptotic access success probability in an equivalent co-located massive MIMO network for analytical comparison. Simulation results verify our analysis and confirm the performance superiority of CF massive MIMO in supporting the GF transmission.

The remainder of this paper is organized as follows. In Section II, the considered system model and motivation of this work are introduced. In Section III, we present the proposed two-disk model for the GF transmission in CF massive MIMO and explain how the disk radii are determined by considering the channel fading and randomness. In Section IV, performance analysis in terms of access success probability based on the proposed model is detailed and theoretical comparison is also provided between CF and co-located massive MIMO. Simulation results are presented in Section V and the work is concluded in Section VI.

Notation: Boldface lower case symbols represent vectors. $\mathbf{I}$ is the identity matrix. $\|\cdot\|$ denotes the Euclidean norm. $\mathbb{E}_{x}[\cdot]$ denotes the expectation operator with respect to variable $x$.

\section{System Model AND Motivation}

\section{A. System Model}

We consider a CF massive MIMO network deployed over a wide area, where a large number of simple and low-cost APs are uniformly spatially distributed to jointly serve a number of active MTC devices over the shared channel resources in uplink. Active devices are assumed to have a limited transmit power, denoted by $\rho_{\mathrm{t}}$, and are triggered to perform GF random access when new packets are to be transmitted. In a typical GF or two-step random access scheme, each active device transmits a randomly selected preamble from an orthogonal pool of size $L$ in the preamble-transmission phase which is followed by a data packet in the data-transmission 
phase [7], where $L$ mini-slots can be considered for data transmissions. That is, an active device that transmits preamble $l(1 \leq l \leq L)$ can transmit its data packet in the $l$ th mini-slot. Moreover, we assume that the distributed APs are connected to a BS central processing unit (CPU) via an error-free fronthaul and that each AP is equipped with $N$ antennas.

Similar to [23], [28], suppose that the APs are randomly positioned on the two-dimensional Euclidean plane and are distributed according to a homogeneous Poisson point processes (PPPs) $\Phi_{\mathrm{AP}} \in \mathbb{R}^{2}$ with density $\lambda_{\mathrm{AP}}\left[\mathrm{APs} / \mathrm{km}^{2}\right]$. Moreover, in each random access slot, devices become active randomly and independently. The locations of active devices follow a two-dimensional homogeneous PPP $\Phi_{\mathrm{D}} \in \mathbb{R}^{2}$ with density $\lambda_{\mathrm{D}}\left[\right.$ devices $\left./ \mathrm{km}^{2}\right]$. For simplicity, we consider a finite area $\mathcal{A}$ with a size of $|\mathcal{A}|\left[\mathrm{km}^{2}\right]$. Consequently, the numbers of APs and active devices in any specific realization follow the Poisson distributions with an average of $\lambda_{\mathrm{AP}}|\mathcal{A}|$ and $\lambda_{\mathrm{D}}|\mathcal{A}|$, respectively.

CF massive MIMO network is a device-centric network [29]. Since $\lambda_{\mathrm{AP}} \gg \lambda_{\mathrm{D}}$ in a CF massive MIMO scenario, the boundary effect caused by the finite-size network region is limited thanks to the macro diversity, i.e., it is highly likely that devices located at the network boundary have close APs making their received signal distribution similar to network-center devices. Therefore, it is sufficient to utilize the typical device approach in CF massive MIMO [30], which facilitates conducting performance analysis and providing insightful results. Thus, without loss of generality, the typical device is assumed located at the origin, which represents a randomly chosen active device in the network.

\section{B. Channel Model}

We consider that the communication link experiences both small-scale fading and large-scale fading in the channel model. In particular, the channel vector between an AP and a device is given by

$$
\mathbf{h}=\sqrt{\beta} \mathbf{g} \in \mathbb{C}^{N}
$$


where $\beta$ denotes the large-scale fading coefficient and $\mathrm{g} \sim \mathcal{C N}\left(0, \mathbf{I}_{N}\right)$ stands for the Rayleigh small-scale fading vector.

In general, the coefficient $\beta$ includes path loss and shadowing, which can be written as

$$
\beta=l_{\circ} \zeta r^{-\alpha},
$$

where $l_{\mathrm{o}}$ is a path-loss constant, $\zeta$ represents an independent shadowing which follows a normal distribution in the decibel scale, i.e., $\zeta[\mathrm{dB}] \sim \mathcal{N}\left(0, \sigma_{\mathrm{sh}}^{2}\right), r[\mathrm{~km}]$ is the distance between an AP and a device, and $\alpha$ is the path-loss exponent.

In this paper, we follow the large-scale fading model proposed by the 3GPP [31] for an urban micro scenario which is considered to be dominated with non-line of sight (NLOS) communication links. The model is further detailed in Section V.

\section{Preamble Collision and Signal Spatial Sparsity}

In GF random access, a preamble collision (i.e., the case that multiple devices select the same preamble) results in contaminated channel estimation for all the collided devices. Moreover, the collided devices end up transmitting data packets in the same mini-slot, which thus leads to data collision and potential transmission failure. In co-located massive MIMO, the preamble/data collision cannot be resolved no matter where collided devices are spatially located because all the signals are eventually assembled at the centralized BS. However, CF massive MIMO opens up a new avenue for addressing the issue.

Specifically, since the signal of a device to different APs undergoes different levels of largescale fading in CF massive MIMO, only neighboring APs within a communication range of a device have non-negligible channel gains thanks to the macro diversity [23], which leads to the signal spatial sparsity in CF massive MIMO [22]. The salient feature is that when only a set of neighboring APs are employed to serve each active device and the sets of APs for different located devices are different or partially overlapped, the impact of preamble/data collision 
among devices could be significantly suppressed at certain serving APs [29], which virtually resolves the preamble collision for collided devices and results in a significantly improved access performance [24] [32].

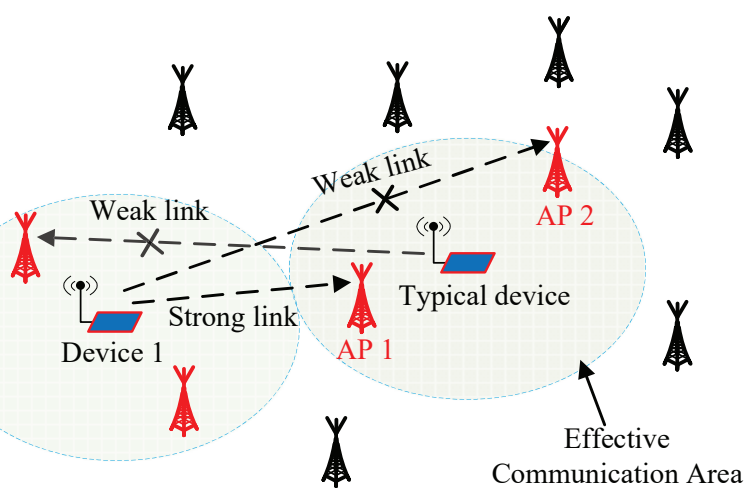

Fig. 1. An example of two-device preamble collision in CF massive MIMO network.

To better explain the advantage of CF massive MIMO, a simplified example of two-device preamble collision is illustrated in Fig. 1. In this example, within a predetermined effective communication area of the typical device, there exist two serving APs, i.e., AP 1 and AP 2, each of which is located close to the typical device. As a result, both the APs are capable of receiving high enough powers from the typical device and attempt to decode the data. The access of the typical device is deemed successful as long as any of the APs can successfully decode its data. In the considered preamble-collision case, we assume that another device, dubbed as device 1, selects the same preamble as the typical device. As a result, device 1's signal acts as interference to the typical device's signal at the both the APs. However, as illustrated in the figure, since device 1 is located close to AP 1 while distant from AP 2, the strengths of interference in the preamble and data domains from device 1 are negligible at AP 2 compared to the strong predominant signal from the typical device. Thus, although AP 1 may fail to decode the desired data due to non-negligible interference from device 1, AP 2 can still decode it successfully thanks to the features of macro diversity and signal spatial sparsity. Similarly, device 1's data could also be successfully decoded by its serving APs in the vicinity. 
The above favorable example gives a glimpse of how CF massive MIMO can be exploited to resolve preamble collisions and support contention-based GF transmission. Nevertheless, in a realistic CF network deployment, the random nature of wireless channels and spatial randomness with respect to the locations and numbers of the APs and active devices have direct impacts on preamble collisions and interference distributions in the GF transmission, which thus need to be taken into account in the performance analysis. To the authors' knowledge, the literature still lacks an effective framework that analytically models and provides a reasonable evaluation on the performance of the GF transmission in CF massive MIMO networks. For this reason, in this work we design a new analytic model oriented to characterize the GF transmission in CF massive MIMO and based on which, a tractable analysis incorporating the stochastic nature of the network is conducted to evaluate and identify the realistic potentials of CF massive MIMO.

\section{Proposed Two-Disk Model in CF Massive MiMO}

To facilitate analysis and characterize the GF transmission in CF massive MIMO, we propose a geometric model in this section, which is underpinned by the distinctive features of CF massive MIMO as identified in Section II-C.

\section{A. Proposed Two-Disk Model}

In particular, we develop a two-disk model for the GF transmission as illustrated in Fig. 2, where one disk centered by the typical device is used to approximate the serving area for its uplink transmission, and the other one centered by an arbitrary AP represents the receiving coverage of the AP, which is utilized to characterize the impact of interference from other active devices that select the same preamble as the typical device in GF transmissions.

As explained in Section II-C, since only neighboring APs of a device have non-negligible channel gains in CF massive MIMO, the effective communication area of the typical device can be approximated by a disk with a radius of $R_{\mathrm{o}}[\mathrm{km}]$, which can also be considered a serving area for the typical device in the uplink transmission, i.e., only the APs within the area are 


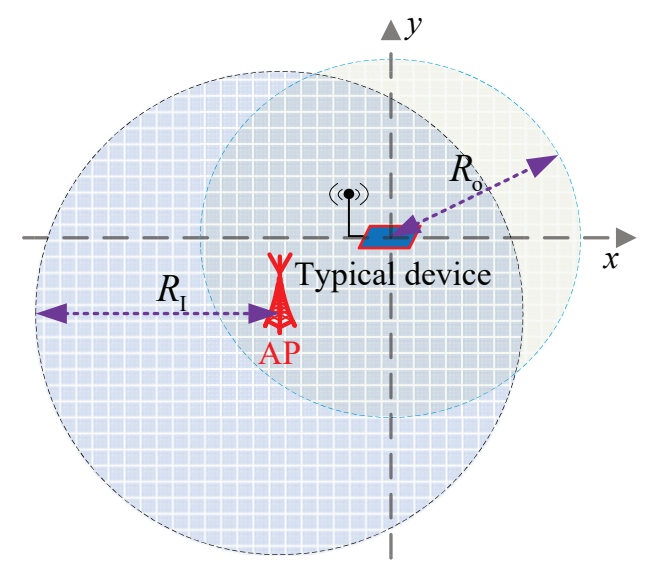

Fig. 2. Illustration of proposed two-disk model in CF massive MIMO network.

employed by the BS CPU to serve the typical device and decode the data locally. As these serving APs are usually located close to the typical device, for simplicity, we assume that each of them can achieve a high enough signal-to-noise ratio (SNR) and accurate channel estimation to successfully decode the data when no preamble collision occurs. Thus, for a given transmit power $\rho_{\mathrm{t}}, R_{\mathrm{o}}$ can be determined by a predetermined SNR threshold, denoted by $\gamma_{\mathrm{o}}$, which should be sufficiently large so that successful data decoding can be guaranteed.

On the other hand, the receiving coverage of an AP is approximately modeled by a disk with a radius of $R_{\mathrm{I}}[\mathrm{km}]\left(R_{\mathrm{I}}>R_{\mathrm{O}}{ }^{1}\right)$. Specifically, considering an arbitrary serving AP of the typical device, if the collided devices (i.e., the devices select the same preamble as the typical device) are located outside the receiving coverage of the AP, it is assumed that their signal strengths are negligible at the AP compared to that of the typical device. Thus, there is no collision effect and the typical device' data can be still successfully decoded in this case. On the other hand, if any of the collided devices is located inside the receiving coverage of the AP, for analysis tractability, we ignore potential capture effects and simply assume that the received signals from the collided and typical devices at the AP result in non-negligible interference to each other,

\footnotetext{
${ }^{1}$ The radius of this receiving coverage of an $\mathrm{AP}, R_{\mathrm{I}}$, is usually larger than that of the communication range, $R_{\mathrm{O}}$, because although the signal from a device outside the communication range cannot be decoded, it can still be an interfering signal at a sufficient distance (i.e., less than or equal to $R_{\mathrm{I}}$ ).
} 
contaminating channel estimation and making data decoding unsuccessful for any of them (i.e., preamble collision results in data transmission failure). Similarly, for a given transmit power $\rho_{\mathrm{t}}$, $R_{\mathrm{I}}$ can also be determined by a predetermined SNR threshold, $\gamma_{\mathrm{I}}$, which should be smaller than $\gamma_{\mathrm{o}}$

It is important to remark that due to the randomness of both the small-scale fading and shadowing in typical terrestrial telecommunications, as depicted in (2), the practical coverage regions are stochastic in shape. This proves to be quite challenging when network designers are trying to obtain accurate representations of the coverage. Nonetheless, approximating a random coverage region by using a uniform disk is a widely used technique adopted in existing works, e.g., [33], which helps provide tractable analysis and insightful results.

In the next subsection, we detail how to empirically obtain the values of the disk radii, i.e., $R_{\mathrm{o}}$ and $R_{\mathrm{I}}$, for given thresholds $\gamma_{\mathrm{o}}$ and $\gamma_{\mathrm{I}}$, respectively.

\section{B. Determining $R_{\mathrm{o}}$ and $R_{\mathrm{I}}$}

In this paper, we determine $R_{\mathrm{o}}$ and $R_{\mathrm{I}}$ by focusing on the $99 \%$ confidence level that the SNRs at an AP are no lower than $\gamma_{\mathrm{o}}$ and $\gamma_{\mathrm{I}}$, respectively.

Based on (1) and (2), for given transmit power $\rho_{\mathrm{t}}$ and distance $r$ between an AP and a device, the received power at the AP can be obtained as follows

$$
\rho_{\mathrm{r}}=\rho_{\mathrm{t}}\|\mathbf{h}\|^{2}=\rho_{\mathrm{t}} l_{\mathrm{o}} G \zeta r^{-\alpha}
$$

where $G=\|\mathbf{g}\|^{2}$ denotes the accumulated power amplitude obtained from different small-scale fading components received by the different $N$ antennas at the AP. Here, $G$ follows a Gamma distribution with a probability density function, $f_{G}(z)=\frac{z^{N-1}}{\Gamma(N)} \exp (-z)$, where $\Gamma(\cdot)$ is the complete Gamma function.

Consequently, the resulting SNR at distance $r$ is obtained as the ratio of the received power to the average noise power $W$, which is a random variable that depends on the two random 
variables (of small-scale fading and shadowing). Accordingly, the probability for the SNR at distance $r$ to be no lower than a threshold $\gamma$ is derived as follows

$$
P(\operatorname{SNR}(r) \geq \gamma)=\int_{0}^{\infty}\left[1-F_{\zeta}\left(\frac{\gamma W r^{\alpha}}{\rho_{\mathrm{t}} z l_{\mathrm{o}}}\right)\right] f_{G}(z) \mathrm{d} z
$$

where $F_{\zeta}(x)$ represents the log-normal cumulative distribution function, i.e., $F_{\zeta}(x)=\frac{1}{2}+$ $\frac{1}{2} \operatorname{erf}\left(-\frac{\ln x}{\sqrt{2} \sigma_{\mathrm{sh}}}\right)$. For the detailed derivation, see Appendix A.

Since at distance $R_{\mathrm{o}}$ a $99 \%$ confidence level that $\operatorname{SNR}\left(R_{\mathrm{o}}\right) \geq \gamma_{\mathrm{o}}$ is expected, $R_{\mathrm{o}}$ can be then determined by

$$
P\left(\operatorname{SNR}\left(R_{\mathrm{o}}\right) \geq \gamma_{\mathrm{o}}\right)=0.99
$$

Similarly, for a given $\gamma_{\mathrm{I}}, R_{\mathrm{I}}$ can be determined as follows

$$
P\left(\operatorname{SNR}\left(R_{\mathrm{I}}\right) \geq \gamma_{\mathrm{I}}\right)=0.99
$$

From (4), we see that $P(\operatorname{SNR}(r) \geq \gamma)$ decreases with $r$. Thus, with (5) and (6), both $R_{\mathrm{o}}$ and $R_{\mathrm{I}}$ can be simply obtained by using well-known root-finding algorithms such as the bi-section method. Note that in practice the values of the predetermined SNR thresholds $\gamma_{\mathrm{o}}$ and $\gamma_{\mathrm{I}}$ depend on the receiver capabilities at APs.

Furthermore, it is straightforward that a larger $\rho_{\mathrm{t}}$ leads to a larger $R_{\mathrm{o}}$ and $R_{\mathrm{I}}$. Nevertheless, since (5) and (6) are exactly the same equations only with different SNR thresholds, it is expected that $\rho_{\mathrm{t}}$ has little impact on the ratio, $\frac{R_{\mathrm{o}}}{R_{\mathrm{I}}}$.

\section{Analysis of AcCess Success Probability}

To further understand the performance of the typical device under the proposed two-disk model, we consider the access success probability as a performance metric in this paper. In this section, based on the proposed two-disk model, we derive an approximated closed-form expression for the access success probability of the typical device by taking the network randomness 
into account and provide an indicative analysis by comparing it to the co-located massive MIMO network under the same setup.

\section{A. Access Success Probability with Two-Disk Model}

Letting the access success probability in the considered CF network be $P_{\mathrm{s}}^{\mathrm{CF}}$, we have

$$
P_{\mathrm{s}}^{\mathrm{CF}}=1-P_{\mathrm{f}}^{\mathrm{CF}}
$$

where $P_{\mathrm{f}}^{\mathrm{CF}}$ is the access failure or outage probability.

Based on the proposed two-disk model, the condition resulting in data transmission failure of the typical device at a serving AP, if it exists, occurs if 1) there exist other active devices selecting the same preamble as the typical device and 2) at least one of these collided devices is located within the receiving coverage of the serving AP. Note that if there are multiple APs serving the typical device, then its data transmission is deemed failed only when all these APs fail to decode the data.

As a result, the access access probability of the typical device can be derived as follows:

$$
P_{\mathrm{s}}^{\mathrm{CF}}=1-\sum_{d=0}^{\infty} \hat{P}\left(d \mid \lambda_{\mathrm{D}}, L\right) \sum_{p=0}^{\infty} \bar{P}\left(p \mid \lambda_{\mathrm{AP}}, R_{\mathrm{o}}\right) \tilde{P}\left(p \mid d, R_{\mathrm{I}}\right)
$$

where $\hat{P}\left(d \mid \lambda_{\mathrm{D}}, L\right)$ represents the probability of exactly $d$ active devices selecting the same preamble as the typical device in area $\mathcal{A}$ for a given $\lambda_{\mathrm{D}}$ and $L, \bar{P}\left(p \mid \lambda_{\mathrm{AP}}, R_{\mathrm{o}}\right)$ represents the probability that exactly $p$ APs are located within the serving area for the typical device for a given $\lambda_{\mathrm{AP}}$ and $R_{\mathrm{o}}$, and $\tilde{P}\left(p \mid d, R_{\mathrm{I}}\right)$ represents the expected probability that all the $p$ serving APs cannot successfully decode the data of the typical device due to the preamble collision for given $d$ collided devices and $R_{\mathrm{I}}$ in area $\mathcal{A}$. The three probabilities can be derived as follows.

Since the active devices follow an independent and homogeneous PPP, according to the thinning process [34], the number of active devices selecting the same preamble as the typical device follows the Poisson distribution with a mean of $\frac{\lambda_{\mathrm{D}}|\mathcal{A}|}{L}$ in area $\mathcal{A}$. Thus, $\hat{P}\left(d \mid \lambda_{\mathrm{D}}, L\right)$ is 
obtained as

$$
\hat{P}\left(d \mid \lambda_{\mathrm{D}}, L\right)=\frac{1}{d !}\left(\frac{\lambda_{\mathrm{D}}|\mathcal{A}|}{L}\right)^{d} \exp \left(-\frac{\lambda_{\mathrm{D}}|\mathcal{A}|}{L}\right) .
$$

In addition, in the serving disk with a radius of $R_{\mathrm{o}}$, the number of serving APs follows the Poisson distribution with a mean of $\lambda_{\mathrm{AP}} \pi R_{\mathrm{o}}^{2}$. As a result, $\bar{P}\left(p \mid \lambda_{\mathrm{AP}}, R_{\mathrm{o}}\right)$ is given by

$$
\bar{P}\left(p \mid \lambda_{\mathrm{AP}}, R_{\mathrm{o}}\right)=\frac{\left(\lambda_{\mathrm{AP}} \pi R_{\mathrm{o}}^{2}\right)^{p}}{p !} \exp \left(-\lambda_{\mathrm{AP}} \pi R_{\mathrm{o}}^{2}\right)
$$

On the other hand, the spatial randomness with respect to the locations of the APs and active devices makes it difficult to derive an exact closed-form expression for the expected probability $\tilde{P}\left(p \mid d, R_{\mathrm{I}}\right)$. To provide an approximation, we start with a hypothetical case, where the receiving coverage of the serving APs for the typical device is assumed independent to each other.

In particular, for any serving $\mathrm{AP}$ among $p$, the probability that at least one of $d$ collided devices in area $\mathcal{A}$ is located within its receiving coverage a radius of $R_{\mathrm{I}}$ is given by

$$
\tilde{P}\left(1 \mid d, R_{\mathrm{I}}\right)=1-\left(1-\frac{\pi R_{\mathrm{I}}^{2}}{|\mathcal{A}|}\right)^{d} \text {. }
$$

In the hypothetical case, treating each serving $\mathrm{AP}$ independent, $\tilde{P}\left(p \mid d, R_{\mathrm{I}}\right)$ is equivalent to $\tilde{P}\left(1 \mid d, R_{\mathrm{I}}\right)$ raised to the power of $p$, i.e.,

$$
\tilde{P}\left(p \mid d, R_{\mathrm{I}}\right)=\left(\tilde{P}\left(1 \mid d, R_{\mathrm{I}}\right)\right)^{p}=\left(1-\left(1-\frac{\pi R_{\mathrm{I}}^{2}}{|\mathcal{A}|}\right)^{d}\right)^{p}
$$

where the exponent $p$ can be interpreted as the ratio of the total receiving coverage by $p$ independent serving APs, i.e., $p \pi R_{\mathrm{I}}^{2}$, to that by a single $\mathrm{AP}$, i.e., $\pi R_{\mathrm{I}}^{2}$, which is formulated as

$$
p=\frac{p \pi R_{\mathrm{I}}^{2}}{\pi R_{\mathrm{I}}^{2}}
$$

Substituting (9), (10), and (12) into (8), we can obtain the access success probability in the hypothetical case. However, in practical case, since all the serving APs are randomly located in 
the disk with a radius of $R_{\mathrm{o}}$ and $R_{\mathrm{I}}>R_{\mathrm{o}}$, their receiving coverage inevitably has overlapping areas, which may cover the same collided devices. Obviously, the hypothetical case is too ideal to be attainable and the practical exponent in (12) cannot be $p$ for $p>1$. Hence, simply using $\left(1-\left(1-\frac{\pi R_{\mathrm{I}}^{2}}{|\mathcal{A}|}\right)^{d}\right)^{p}$ to represent practical $\tilde{P}\left(p \mid d, R_{\mathrm{I}}\right)$ in (8) can be way off the mark when $p$ is large.

To obtain a more accurate approximation for $\tilde{P}\left(p \mid d, R_{\mathrm{I}}\right)$, we consider practical cases and modify (12) based on the insights gained from the hypothetical case as follows. Specifically, in practical cases the exponent in (12) can be analogously interpreted as the ratio of the total receiving coverage by all $p$ serving APs to that by a single AP. We denote by $S_{p}$ the total receiving coverage by all $p$ serving APs and $\xi_{p}$ the exponent in practical cases. Then, we have

$$
\xi_{p}=\frac{S_{p}}{\pi R_{\mathrm{I}}^{2}}
$$

Note that in practical cases, for a given $p$, both $S_{p}$ and $\xi_{p}$ are variables whose values can change depending on the relative locations of serving APs. Keeping this in mind and replacing $p$ by $\xi_{p}$ in (12), $\tilde{P}\left(p \mid d, R_{\mathrm{I}}\right)$ is rewritten as

$$
\tilde{P}\left(p \mid d, R_{\mathrm{I}}\right)=\mathbb{E}_{\xi_{p}}\left[\left(1-\left(1-\frac{\pi R_{\mathrm{I}}^{2}}{|\mathcal{A}|}\right)^{d}\right)^{\xi_{p}}\right] .
$$

To get rid of the expectation operation in (15), we consider a lower bound $\underline{\tilde{P}}\left(p \mid d, R_{\mathrm{I}}\right)$ as an approximation. Denote by $\bar{S}_{p}$ and $\bar{\xi}_{p}=\frac{\bar{S}_{p}}{\pi R_{\mathrm{I}}^{2}}$ the largest $S_{p}$ and $\xi_{p}$ that can be achieved for a given $p$, respectively. Then, we have

$$
\begin{aligned}
\underline{\tilde{P}}\left(p \mid d, R_{\mathrm{I}}\right) & =\left(1-\left(1-\frac{\pi R_{\mathrm{I}}^{2}}{|\mathcal{A}|}\right)^{d}\right)^{\bar{\xi}_{p}} \\
& \leq \tilde{P}\left(p \mid d, R_{\mathrm{I}}\right) .
\end{aligned}
$$

To further obtain $\underline{\tilde{P}}\left(p \mid d, R_{\mathrm{I}}\right)$, we need to calculate $\bar{\xi}_{p}$ for each $p$, which is unfortunately computationally infeasible since $p$ is ranging from 0 to $\infty$. Herein, to avoid heavy computation, instead 
of calculating all $\bar{\xi}_{p}$, we use a feasible approximation that only calculates $\bar{\xi}_{p}$ for $p$ up to 3 and uses $\bar{\xi}_{3}$ to approximate $\bar{\xi}_{p}$ for $p \geq 4$. This simplification is conducted based on the fact that since the locations of all $p$ serving APs are restricted in the serving disk with a radius of $R_{\mathrm{o}}, \bar{S}_{p}$ will exhibit a slow increase as $p$ increases and asymptotically approach a constant. This indicates that when $p$ is sufficiently large further increasing it only brings limited benefit in terms of spatial diversity for the typical device's data transmission. Therefore, $\bar{\xi}_{p}$ in this approximation is given by

$$
\bar{\xi}_{p}= \begin{cases}0 & \text { if } p=0 \\ 1 & \text { if } p=1 \\ \bar{\xi}_{2} & \text { if } p=2 \\ \bar{\xi}_{3} & \text { if } p \geq 3\end{cases}
$$

where $\bar{\xi}_{2}$ is derived as

$$
\bar{\xi}_{2}=2-\frac{\theta-\sin \theta}{\pi}
$$

where $\theta=2 \arccos \frac{R_{\mathrm{o}}}{R_{\mathrm{I}}}$. And $\bar{\xi}_{3}$ is derived as

$$
\bar{\xi}_{3}=3-\frac{3 \theta_{1}-3 \sin \theta_{1}}{\pi}+\frac{6 \theta_{2} R_{\mathrm{I}}^{2}-6 a \sqrt{R_{\mathrm{I}}^{2}-\frac{a^{2}}{4}}+\sqrt{3} a^{2}}{4 \pi R_{\mathrm{I}}^{2}},
$$

where $\theta_{1}=2 \arccos \frac{\sqrt{3} R_{\mathrm{o}}}{2 R_{\mathrm{I}}}, a=\sqrt{3}\left|\sqrt{R_{\mathrm{I}}^{2}-\frac{3 R_{\mathrm{o}}^{2}}{4}}-\frac{R_{\mathrm{o}}}{2}\right|$, and $\theta_{2}=2 \arcsin \frac{a}{2 R_{\mathrm{I}}}$. For detailed derivations for $\bar{\xi}_{2}$ and $\bar{\xi}_{3}$, see Appendix B.

Substituting (9), (10), and (16) with (17) into (8), we obtain the approximated mathematical expression for $P_{\mathrm{s}}^{\mathrm{CF}}$.

\section{B. Discussions}

The above theoretical analysis provides insights into the performance behaviors of GF transmission with respect to key system parameters in the context of CF massive MIMO, which are discussed as follows. 
From (10), we see that the average number of serving APs for the typical device is $\lambda_{\mathrm{AP}} \pi R_{\mathrm{o}}^{2}$. Since the second summation in (8) is mainly dominated by the term of $p=\lambda_{\mathrm{AP}} \pi R_{\mathrm{o}}^{2}$, and $\bar{P}(p=$ $\lambda_{\mathrm{AP}} \pi R_{\mathrm{o}}^{2} \mid \lambda_{\mathrm{AP}}, R_{\mathrm{o}}$ ) decreases as $\lambda_{\mathrm{AP}}$ increases (but with slower decreasing rate), it indicates that $P_{\mathrm{s}}^{\mathrm{CF}}$ increases with $\lambda_{\mathrm{AP}}$ (with slower increasing rate). The physical intuition behind this is that a larger $\lambda_{\mathrm{AP}}$ means that on average more APs will be located in the disk with a radius of $R_{\mathrm{o}}$ and serve the typical device, which thus increases the spatial diversity gain and $P_{\mathrm{s}}^{\mathrm{CF}}$ as a result. However, when the average number of serving APs becomes sufficiently large within the disk, as indicated in Section IV-A, the spatial diversity gain will get saturated. Thus, further increasing it only brings insignificant benefit in the increase of $P_{\mathrm{s}}^{\mathrm{CF}}$. In addition, since a smaller $\lambda_{\mathrm{D}}$ or larger $L$ leads to a lower preamble collision at the serving APs, $P_{\mathrm{s}}^{\mathrm{CF}}$ can be improved as a result.

Besides, as indicated in Section III-B, with predetermined SNR thresholds, $R_{\mathrm{o}}$ and $R_{\mathrm{I}}$ are determined by the transmit power $\rho_{\mathrm{t}}$, and a larger $\rho_{\mathrm{t}}$ leads to a larger $R_{\mathrm{o}}$ and $R_{\mathrm{I}}$ but almost fixed ratio of $\frac{R_{\mathrm{o}}}{R_{\mathrm{I}}}$. Thus, $\bar{\xi}_{p}$ can be treated independently of $\rho_{\mathrm{t}}$. From (10), it is also evident that $R_{\mathrm{o}}$ has the same effect on $P_{\mathrm{s}}^{\mathrm{CF}}$ as $\lambda_{\mathrm{AP}}$. Thus, $P_{\mathrm{s}}^{\mathrm{CF}}$ also increases with $R_{\mathrm{o}}$ but with slower increasing rate. On the other hand, a larger $R_{\mathrm{I}}$ indicates that the collided devices are more likely located within the receiving coverage of the serving APs, as indicated in (15), leading to higher probabilities of data transmission failure due to the preamble collision. Therefore, a larger $\rho_{\mathrm{t}}$ does not necessarily lead to a better access performance in CF massive MIMO network. In fact, it is important to balance the trade-off between the above contradicting factors and the optimal $\rho_{\mathrm{t}}$ needs to be determined for given system setup and network deployment.

Since the BS CPU can calculate $P_{\mathrm{s}}^{\mathrm{CF}}$ by using the derived mathematical approximations, the optimal $\rho_{\mathrm{t}}$ can be simply determined by finding the one with the highest $P_{\mathrm{s}}^{\mathrm{CF}}$ in a given transmit power range or set. In practice, with the determined optimal transmit power, the BS CPU can broadcast it to the devices via a system information block (SIB) prior to the start of the random access procedure. 


\section{Comparison to Co-located Massive MIMO}

The performance of CF massive MIMO can be compared to that of co-located massive MIMO, which is a BS-centric network where all the serving antennas are located at a centralized BS to serve the devices in area $\mathcal{A}$.

To make a fair and simple comparison, we assume that a centralized BS is located at the origin and is equipped with $N \lambda_{\mathrm{AP}}|\mathcal{A}|$ antennas, while the typical device is randomly located in the area. A single-disk model for the GF transmission is considered in the co-located network architecture, where the receiving coverage of the BS is approximated by a disk with a radius of $R_{\mathrm{I}}^{\mathrm{Co}}[\mathrm{km}]$, which can be seen as an extension of the receiving coverage of an AP in CF massive MIMO when the receiver at the AP has an additional array gain of $\lambda_{\mathrm{AP}}|\mathcal{A}|$. Therefore, based on Section III-B, $R_{\mathrm{I}}^{\mathrm{Co}}$ can also be determined by focusing on the $99 \%$ confidence level that the corresponding SNR at the centralized BS is no lower than $\gamma_{\mathrm{I}}$. Note that in the CF network, determining $R_{\mathrm{o}}$ and $R_{\mathrm{I}}$ is independent of $\lambda_{\mathrm{AP}}$. However, in the co-located network, it is obvious that a larger $\lambda_{\mathrm{AP}}$ leads to a larger $R_{\mathrm{I}}^{\mathrm{Co}}$.

Similar to what we assume in the disk with a radius of $R_{\mathrm{I}}$, in the extended disk, we ignore potential capture effects and assume that 1) preamble collision results in contaminated channel estimation and thus data transmission failure for any of collided devices that are inside the disk;

2) the strengths of signals from outside of the disk are negligible and data cannot be decoded by the centralized BS; and 3) when an active device is inside the disk and has a collision-free transmission, its data can be successfully decoded.

As $R_{\mathrm{I}}^{\mathrm{C}}$ increases with $\lambda_{\mathrm{AP}}$, when $\lambda_{\mathrm{AP}}$ becomes significantly large, we see that $R_{\mathrm{I}}^{\mathrm{C}}$ can be large enough so that the receiving coverage of the centralized BS can cover the entire area $\mathcal{A}$. As a result, similar to the two-disk model and as presented in (9), the number of collided devices selecting the same preamble as the typical device follows the Poisson distribution with a mean of $\frac{\lambda_{\mathrm{D}}|\mathcal{A}|}{L}$ in area $\mathcal{A}$. Thus, in the context of co-located massive MIMO, the asymptotic access 
success probability is given by

$$
P_{\mathrm{s}}^{\mathrm{Co}}=\hat{P}\left(d=0 \mid \lambda_{\mathrm{D}}, L\right)=\exp \left(-\frac{\lambda_{\mathrm{D}}|\mathcal{A}|}{L}\right)
$$

which is independent of $\lambda_{\mathrm{AP}}$. This aligns with the findings in [35]. That is, for a given area $\mathcal{A}$, when the number of serving antennas is sufficiently large, the performance of GF transmission in co-located massive MIMO is mainly dominated by $\lambda_{\mathrm{D}}$ and $L$.

By comparing (20) to (8), the following lemma can be obtained.

Lemma 1: In the asymptotic region, i.e., $\lambda_{\mathrm{AP}} \rightarrow \infty$, we have $P_{\mathrm{s}}^{\mathrm{CF}}>P_{\mathrm{s}}^{\mathrm{Co}}$.

Proof: With (20), $P_{\mathrm{s}}^{\mathrm{Co}}$ can be rewritten as

$$
P_{\mathrm{s}}^{\mathrm{Co}}=\hat{P}\left(d=0 \mid \lambda_{\mathrm{D}}, L\right)=1-\sum_{d=1}^{\infty} \hat{P}\left(d \mid \lambda_{\mathrm{D}}, L\right) .
$$

For $P_{\mathrm{s}}^{\mathrm{CF}}$, we have $\tilde{P}\left(p \mid d=0, R_{\mathrm{I}}\right)=0$ for $p \geq 1$ and $\bar{P}\left(p=0 \mid \lambda_{\mathrm{AP}}, R_{\mathrm{o}}\right) \rightarrow 0$ as $\lambda_{\mathrm{AP}} \rightarrow \infty$, it thus can be further written as

$$
P_{\mathrm{s}}^{\mathrm{CF}}=1-\sum_{d=1}^{\infty} \hat{P}\left(d \mid \lambda_{\mathrm{D}}, L\right) \sum_{p=1}^{\infty} \bar{P}\left(p \mid \lambda_{\mathrm{AP}}, R_{\mathrm{o}}\right) \tilde{P}\left(p \mid d, R_{\mathrm{I}}\right)
$$

Since $\sum_{p=1}^{\infty} \bar{P}\left(p \mid \lambda_{\mathrm{AP}}, R_{\mathrm{o}}\right) \tilde{P}\left(p \mid d, R_{\mathrm{I}}\right)<1$, we then have $P_{\mathrm{s}}^{\mathrm{CF}}>P_{\mathrm{s}}^{\mathrm{Co}}$, which completes the proof.

As in the $\mathrm{CF}$ massive MIMO scenario, $\lambda_{\mathrm{AP}}$ is usually very large. Lemma 1 indicates that the devices in CF massive MIMO can achieve a better access performance than co-located massive MIMO. Intuitively, this is sensible since all the active devices' signals are received at the centralized BS in co-located massive MIMO, the preamble collision cannot be resolved and thus the data of collided devices cannot be decoded successfully. However, as explained in Sections II-C and III, this is not the case in CF massive MIMO, i.e., even when a preamble collision occurs, the distributed serving APs are still capable of decoding the devices' data thanks to the signal spatial sparsity and random geographic distributions of devices. 


\section{Simulation Results}

In this section, the simulation results are presented to show the GF transmission performance with the proposed two-disk model and verify the derivations obtained in the previous section. To depict the performance of a realistic network, we capitalize on [31] to define the network channel parameters. In particular, the path-loss exponent $\alpha=3.67$, the shadowing standard deviation $\sigma_{\mathrm{sh}}=4$, and the path-loss constant is set to $l_{\mathrm{o}}[\mathrm{dB}]=-3 \alpha-22.7-26 \log _{10}\left(f_{\mathrm{c}}\right)$, where $f_{\mathrm{c}}[\mathrm{GHz}]$ is the carrier frequency. The additive thermal noise is assumed to have a power spectral density of $-174 \mathrm{dBm} / \mathrm{Hz}$ while the front-end receiver at each $\mathrm{AP}$ is assumed to have a noise figure of $9 \mathrm{~dB}$ [18]. Thus, the average noise power $W=-92 \mathrm{dBm}$ with a bandwidth of $B=20 \mathrm{MHz}$. In addition, the transmit and receive gains are set to $5 \mathrm{~dB}$ and $2 \mathrm{~dB}$, respectively. For the SNR thresholds, $\gamma_{\mathrm{I}}$ is set to $0 \mathrm{~dB}$ and $\gamma_{\mathrm{o}}$ is set to 6 or $10 \mathrm{~dB}$, which corresponds to different levels of receiver capabilities at each AP. The parameter setups are summarized in Table I. To understand the performance behaviors in CF massive MIMO network and compare it to those in the co-located one, we vary key parameters, i.e. $\rho_{\mathrm{t}}, \lambda_{\mathrm{D}}$, and $\lambda_{\mathrm{AP}}$, to study and verify their impacts on the access success probability.

TABLE I

PARAMETER SETUP

\begin{tabular}{cll}
\hline \hline Symbol & Definition & Value \\
\hline $\mathcal{A}$ & Network area & $1 \mathrm{~km} \times 1 \mathrm{~km}$ \\
$L$ & Preamble pool size & $48[36]$ \\
$N$ & Number of antennas per AP & 2 \\
$f_{\mathrm{c}}$ & Carrier frequency & $2 \mathrm{GHz}$ \\
$\alpha$ & Path-loss exponent & 3.67 \\
$l_{\mathrm{o}}$ & Path-loss constant & $-30.53 \mathrm{~dB}$ \\
$\sigma_{\mathrm{sh}}$ & Shadowing standard deviation & $4 \mathrm{~dB}$ \\
$B$ & Channel bandwidth & $20 \mathrm{MHz}$ \\
$W$ & Average noise power & $-92 \mathrm{dBm}$ \\
$\gamma_{\mathrm{o}}$ & SNR threshold for $R_{\mathrm{o}}$ & $6 \mathrm{or} 10 \mathrm{~dB}$ \\
$\gamma_{\mathrm{I}}$ & SNR threshold for $R_{\mathrm{I}}$ & $0 \mathrm{~dB}$ \\
\hline
\end{tabular}

In Tables II and III, based on Section III-B, we present the values of disk radii, i.e., $R_{\mathrm{o}}$ and $R_{\mathrm{I}}$, defined in the proposed two-disk model for different values of $\rho_{\mathrm{t}}$ and $\gamma_{\mathrm{o}}$. In particular, we 
consider a common transmit power range, i.e., $\rho_{\mathrm{t}}=\{17,20,23,27\}[\mathrm{dBm}]$, for MTC devices. As expected, in each table both the disk radii increases with $\rho_{\mathrm{t}}$ but their ratios are kept almost the same.

TABLE II

DisK RADII VALUES WHEN $\gamma_{\mathrm{o}}=10 \mathrm{DB}$ AND $\gamma_{\mathrm{I}}=0 \mathrm{DB}$

\begin{tabular}{cccc}
\hline \hline$\rho_{\mathrm{t}}[\mathrm{dBm}]$ & $R_{\mathrm{o}}[\mathrm{km}]$ & $R_{\mathrm{I}}[\mathrm{km}]$ & $\frac{R_{\mathrm{o}}}{R_{\mathrm{I}}}$ \\
\hline 17 & 0.054 & 0.102 & 0.529 \\
20 & 0.066 & 0.124 & 0.532 \\
23 & 0.081 & 0.151 & 0.536 \\
27 & 0.104 & 0.195 & 0.533 \\
\hline
\end{tabular}

TABLE III

Disk RAdiI VALUES WHEN $\gamma_{\mathrm{o}}=6$ DB AND $\gamma_{\mathrm{I}}=0 \mathrm{DB}$

\begin{tabular}{cccc}
\hline \hline$\rho_{\mathrm{t}}[\mathrm{dBm}]$ & $R_{\mathrm{o}}[\mathrm{km}]$ & $R_{\mathrm{I}}[\mathrm{km}]$ & $\frac{R_{\mathrm{o}}}{R_{\mathrm{I}}}$ \\
\hline 17 & 0.069 & 0.102 & 0.676 \\
20 & 0.084 & 0.124 & 0.677 \\
23 & 0.103 & 0.151 & 0.682 \\
27 & 0.133 & 0.195 & 0.682 \\
\hline
\end{tabular}

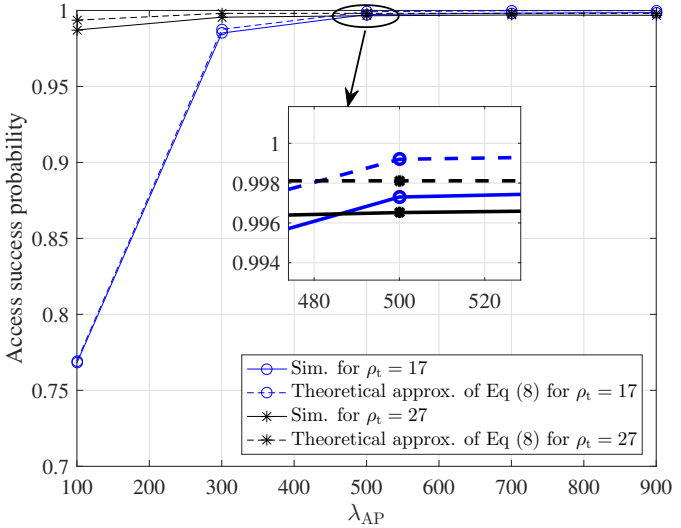

(a) When $\gamma_{\mathrm{o}}=6 \mathrm{~dB}$ and $\gamma_{\mathrm{I}}=0 \mathrm{~dB}$.

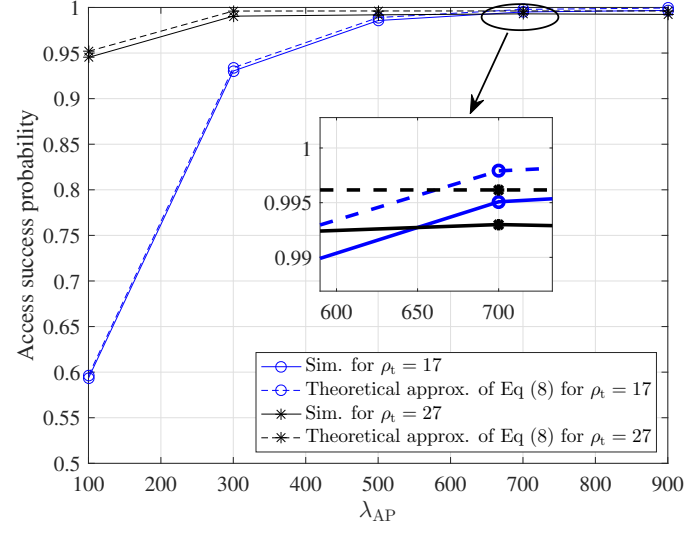

(b) When $\gamma_{\mathrm{o}}=10 \mathrm{~dB}$ and $\gamma_{\mathrm{I}}=0 \mathrm{~dB}$.

Fig. 3. Access success probability as a function of $\lambda_{\mathrm{AP}}$ with different $\rho_{\mathrm{t}}$ at $\lambda_{\mathrm{D}}=10$ in CF massive MIMO.

In Figs. 3 and 4, the access success probabilities in CF massive MIMO are plotted as functions of $\lambda_{\mathrm{AP}}$ at $\lambda_{\mathrm{D}}=10$ and $\lambda_{\mathrm{D}}=30$, respectively. In each figure, two sub-figures are presented with $\gamma_{\mathrm{o}}=6 \mathrm{~dB}$ and $10 \mathrm{~dB}$, respectively, and different values of $\rho_{\mathrm{t}}$ are considered in each sub-figure. 


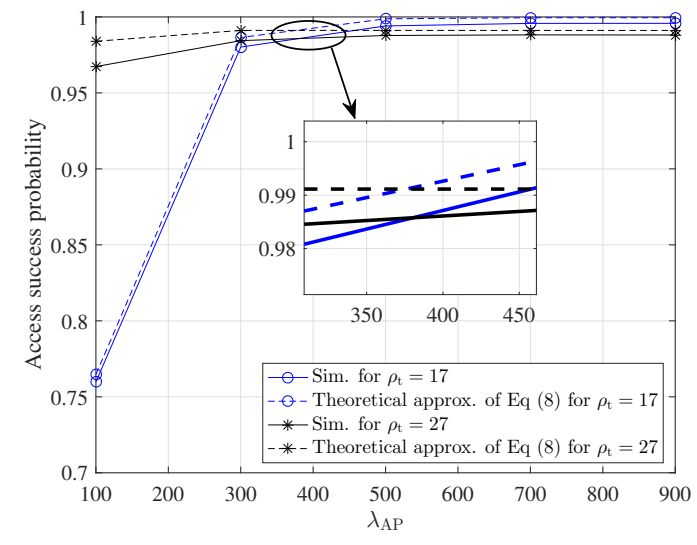

(a) When $\gamma_{\mathrm{o}}=6 \mathrm{~dB}$ and $\gamma_{\mathrm{I}}=0 \mathrm{~dB}$.

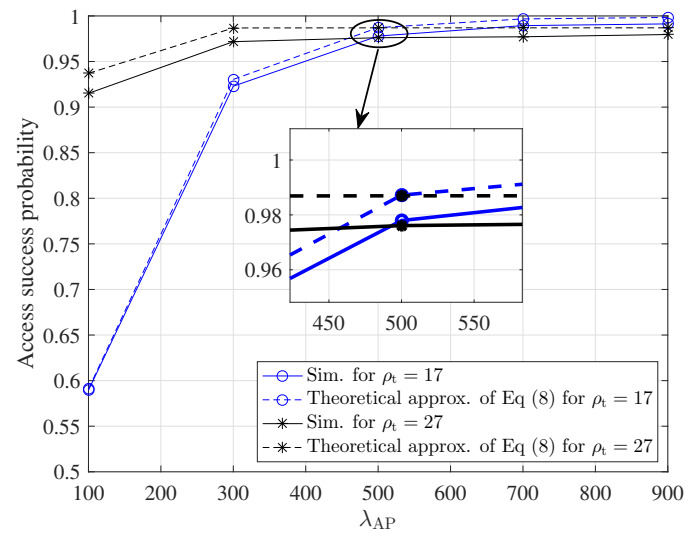

(b) When $\gamma_{\mathrm{o}}=10 \mathrm{~dB}$ and $\gamma_{\mathrm{I}}=0 \mathrm{~dB}$.

Fig. 4. Access success probability as a function of $\lambda_{\mathrm{AP}}$ with different $\rho_{\mathrm{t}}$ at $\lambda_{\mathrm{D}}=30$ in CF massive MIMO.

As shown, the theoretical approximation holds tightly with the simulation results under various system setups. Since a lower $\gamma_{\mathrm{o}}$ leads to a larger $R_{\mathrm{o}}$ and a larger $R_{\mathrm{o}}$ leads to an increased success probability as analyzed in Section IV-B, we see in both the figures that a lower $\gamma_{0}$ results in a higher success probability. Nevertheless, we recall that like $\gamma_{\mathrm{I}}, \gamma_{\mathrm{o}}$ is a predetermined system parameter and a lower $\gamma_{\mathrm{o}}$ indicates that a more capable receiver is required to successfully decode data at APs. In addition, as $\lambda_{\mathrm{AP}}$ becomes larger, we see that the success probability increases and approaches one with a slower increasing rate regardless of $\rho_{\mathrm{t}}$ and $\lambda_{\mathrm{D}}$. This observation also validates our analysis in Section IV-B, which corresponds to that a larger $\lambda_{\mathrm{AP}}$ leads to a larger spatial diversity gain and lower chances of data transmission failure for the typical device. Thus, the success probability can increase. Nonetheless, when $\lambda_{\mathrm{AP}}$ becomes sufficiently large, as indicated in Section IV-A, the spatial diversity gain will get saturated and the increase of the success probability tends to flatten out.

Moreover, as analyzed in Section IV-B, both the figures show that a larger transmit power does not necessarily results in a higher success probability in CF massive MIMO, which in fact highly depends on the values of $\lambda_{\mathrm{AP}}$. For instance, in Fig. 3(b), the success probability at $\rho_{\mathrm{t}}=27$ is significantly higher that that at $\rho_{\mathrm{t}}=17$ when $\lambda_{\mathrm{AP}}=100$. However, when $\lambda_{\mathrm{AP}}>650$, adopting $\rho_{\mathrm{t}}=17$ is able to achieve a better performance instead. This can be explained by the 
fact that when $\lambda_{\mathrm{AP}}$ and $\rho_{\mathrm{t}}$ are both small there may be very few APs in the disk with a radius of $R_{\mathrm{o}}$ to serve the typical device. Thus, adopting higher transmit power is essential to increase the spatial diversity gain in this case. On the other hand, when $\lambda_{\mathrm{AP}}$ is sufficiently large, using a properly low $\rho_{\mathrm{t}}$ can not only provide a decent spatial diversity gain for the typical device, but also lead to a small $R_{\mathrm{I}}$ and thus a low collision probability within the receiving coverage of the serving APs. As a result, a better access performance can be achieved with a low transmit power in this case.

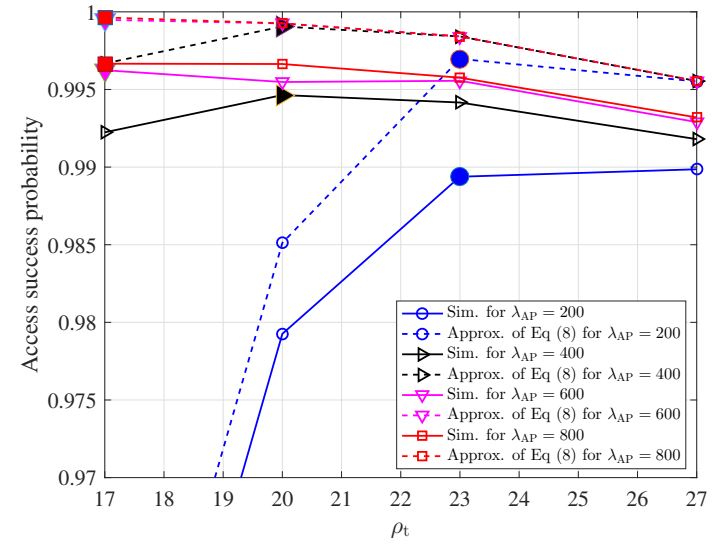

(a) When $\gamma_{\mathrm{o}}=6 \mathrm{~dB}$ and $\gamma_{\mathrm{I}}=0 \mathrm{~dB}$.

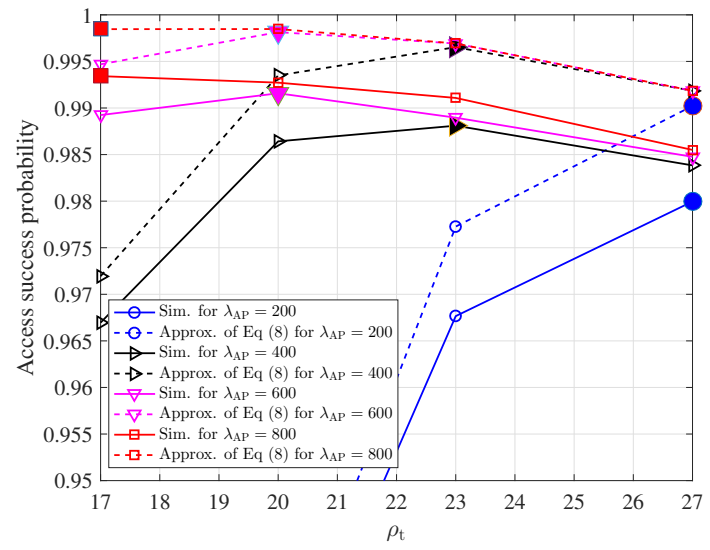

(b) When $\gamma_{\mathrm{o}}=10 \mathrm{~dB}$ and $\gamma_{\mathrm{I}}=0 \mathrm{~dB}$.

Fig. 5. Access success probability as a function of $\rho_{\mathrm{t}}$ with different $\lambda_{\mathrm{AP}}$ at $\lambda_{\mathrm{D}}=20$ in CF massive MIMO.

Fig. 5 illustrates the access success probability by varying $\rho_{\mathrm{t}}$ for different values of $\lambda_{\mathrm{AP}}$ and $\gamma_{\mathrm{o}}$ at $\lambda_{\mathrm{D}}=20$. As we can see, for any given $\lambda_{\mathrm{AP}}$ in each sub-figure, there exists an optimal $\rho_{\mathrm{t}}$ maximizing the success probability, which is represented by the filled marker point in each curve. Evidently, the optimal $\rho_{\mathrm{t}}$ tends to decrease as $\lambda_{\mathrm{AP}}$ increases. These observations further verify the above explanations. Furthermore, it is shown in both the sub-figures that the derived theoretical approximation accurately reflects the performance behaviors with respect to $\rho_{\mathrm{t}}$. Therefore, as explained in Section IV-B, the BS CPU can exploit it to easily find the optimal $\rho_{\mathrm{t}}$ for given system setup and network deployment and broadcast this information to the devices prior to the start of the random access procedure. As such, the devices can achieve the best access performance in terms of access success probability. 
TABLE IV

VALUES OF RADIUS $R_{\mathrm{I}}^{\text {Co }}[\mathrm{KM}]$ FOR Co-LOCATED MASSIVE MIMO

\begin{tabular}{c|ccccc}
\hline \hline $\begin{array}{c}\rho_{\mathrm{t}} \\
{[\mathrm{dBm}]}\end{array}$ & \multicolumn{5}{|c}{$\lambda_{\mathrm{AP}}$} \\
\cline { 2 - 6 } & 100 & 300 & 500 & 700 & 900 \\
\hline 17 & 0.503 & 0.676 & 0.772 & 0.848 & 0.906 \\
27 & 0.944 & 1.251 & 1.463 & 1.597 & 1.693 \\
\hline
\end{tabular}

In the following, we compare the performance in terms of access success probability between CF and co-located massive MIMO in the case of $\gamma_{\mathrm{o}}=6 \mathrm{~dB}$ and $\gamma_{\mathrm{I}}=0 \mathrm{~dB}$. For the co-located massive MIMO scenario, the values of its coverage radius with different $\rho_{\mathrm{t}}$ and $\lambda_{\mathrm{AP}}$ are given

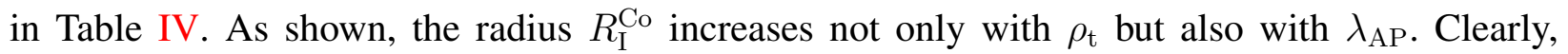
for a fixed $\rho_{\mathrm{t}}$, the entire area $\mathcal{A}$ eventually will be covered by the centralized $\mathrm{BS}$ as long as $\lambda_{\mathrm{AP}}$ becomes sufficiently large. As a result, the asymptotic success probability in (20) will be achieved. This is observed in Fig. 6. From the figure, regardless of $\rho_{\mathrm{t}}$, it is seen that the success probability in co-located massive MIMO approaches the derived asymptotic success probability as $\lambda_{\mathrm{AP}}$ increases. In addition, by comparing the performance between the two different network architectures, it is evident that CF massive MIMO is able to considerably outperform its colocated counterpart and improve the success probability especially for large $\lambda_{\mathrm{AP}}$, which validates Lemma 1. For instance, when $\rho_{\mathrm{t}}=17$ and $\lambda_{\mathrm{AP}}=500$, a close-to-one success probability, i.e., 0.995, can be achieved in CF massive MIMO, while it is only 0.659 in the co-located one. As explained in previous sections, the significant improvements are resulted from the advantage of macro diversity and signal spatial sparsity in CF massive MIMO, which can be exploited to effectively resolve preamble and data collisions for the GF transmission in random access.

To further show the performance superiority of CF massive MIMO in supporting the GF transmission, Fig. 7 depicts the access success probability as a function of $\lambda_{\mathrm{D}}$ at $\rho_{\mathrm{t}}=17$ and $\lambda_{\mathrm{AP}}=500$. Again, our theoretical analysis well matches the simulation results for both $\mathrm{CF}$ and co-located massive MIMO. Since a larger $\lambda_{\mathrm{D}}$ means more active devices on average contending for the shared channel resource in GF random access, both the success probabilities decrease 


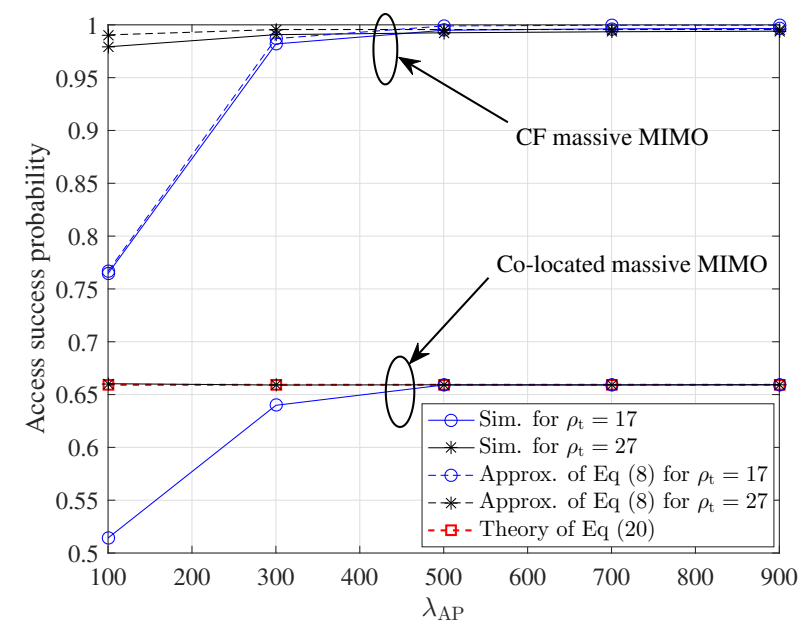

Fig. 6. Access success probability as a function of $\lambda_{\mathrm{AP}}$ with different $\rho_{\mathrm{t}}$ at $\lambda_{\mathrm{D}}=20$ in CF and co-located massive MIMO.

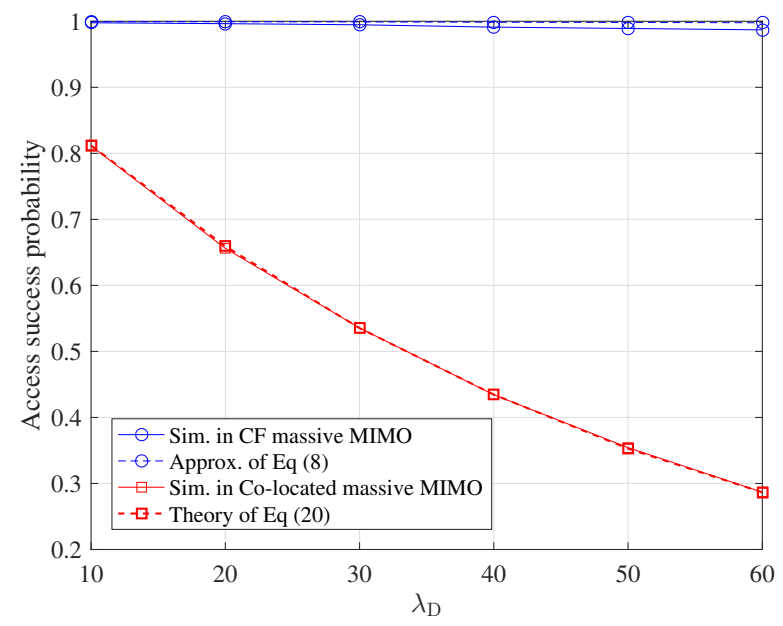

Fig. 7. Access success probability as a function of $\lambda_{\mathrm{D}}$ at $\rho_{\mathrm{t}}=17$ and $\lambda_{\mathrm{AP}}=500$ in CF and co-located massive MIMO.

with $\lambda_{\mathrm{D}}$. However, as observed, the decrease is trivial in CF massive MIMO over a wide range of $\lambda_{\mathrm{D}}$ and its success probability still keeps close to one even when $\lambda_{\mathrm{D}}$ is large, e.g., $\lambda_{\mathrm{D}}=60$. On the other hand, we see that the performance in co-located massive MIMO is degraded rapidly, dropping from 0.811 at $\lambda_{\mathrm{D}}=10$ to 0.287 at $\lambda_{\mathrm{D}}=60$. These observations demonstrate that unlike co-located massive MIMO, CF massive MIMO is able to provide robust performance in terms of access reliability against increased density of active devices in the network, which is highly desired in crowd MTC scenarios. 


\section{CONCLUSIONS AND REMARKS}

In this paper, the performance of GF random access was evaluated under a realistic network deployment scenario of CF massive MIMO, where the spatial randomness regarding the locations of APs and devices are taken into consideration. By capitalizing on the distinctive CF network architecture and features, we first developed a new two-disk based analytic model for the GF transmission in $\mathrm{CF}$ massive MIMO. Based on the proposed two-disk model, we then focused on the performance evaluation and derived an approximated closed-form expression for the access success probability by using techniques from stochastic geometry, which helps extract some interesting insights into the performance behaviors with respect to different key system parameters. To show the performance superiority of CF massive MIMO to its co-located counterpart, we used an analogous single-disk model to characterize the GF transmission in co-located massive MIMO and provided an analytical comparison between the two different architectures. Simulation results verified our analysis and confirmed that there exists an optimal transmit power that maximizes the access success probability in a specific CF network deployment. It has also been demonstrated that CF massive MIMO can significantly outperform co-located massive MIMO in terms of access success probability and provide robust performance against increased access density, which well suits to crowd scenarios.

As a preliminary study, this work considered a fully decentralized CF implementation in signal processing, i.e., serving APs decode data locally without cooperation, which is highly desirable from the perspective of fronthaul overhead and practical deployment. Nevertheless, to further improve the performance, a hybrid CF implementation scheme can be considered. That is, if all the serving APs fail to decode the data locally, they can further forward received data information to the BS CPU, which can then perform a centralized joint data decoding. Therefore, a potential extension of this work would be to apply the hybrid CF implementation scheme into the proposed two-disk model for performance analysis. 


\section{APPENDIX A}

\section{CAlculating the SNR Probability}

The SNR is the ratio of the received power to the average noise power at the receiver. By using (3), the $\operatorname{SNR}$ at a distance $r$ is obtained as $\operatorname{SNR}(r)=\frac{\rho_{\mathrm{r}}}{W}=\frac{\rho_{\mathrm{t}} l_{\mathrm{o}} G \zeta r^{-\alpha}}{W}$. Since the $\mathrm{SNR}$ is not deterministic, we derive the probability that is no lower than a threshold as follows

$$
\begin{aligned}
P(\operatorname{SNR}(r) \geq \gamma) & \stackrel{(\mathrm{a})}{=} P\left(\zeta \geq \frac{\gamma W r^{\alpha}}{\rho_{\mathrm{t}} G l_{\mathrm{o}}}\right) \\
& \stackrel{(\mathrm{b})}{=} \mathbb{E}_{G}\left[1-F_{\zeta}\left(\frac{\gamma W r^{\alpha}}{\rho_{\mathrm{t}} z l_{\mathrm{o}}}\right)\right] \\
& =\int_{0}^{\infty}\left[1-F_{\zeta}\left(\frac{\gamma W r^{\alpha}}{\rho_{\mathrm{t}} z l_{\mathrm{o}}}\right)\right] f_{G}(z) \mathrm{d} z,
\end{aligned}
$$

where (a) stems from the fact that the shadowing random variable is independent from the small-scale fading and (b) stems from the definition of the cumulative distribution function.

\section{APPENDIX B \\ CALCULATING $\bar{\xi}_{2}$ AND $\bar{\xi}_{3}$}

A. calculating $\bar{\xi}_{2}$

Recall that $\bar{\xi}_{2}$ is achieved when $p=2$ serving APs located in the disk with a radius of $R_{\mathrm{o}}$ have the largest total receiving coverage $\bar{S}_{2}$. Obviously, this happens when the two APs are located at the edge of the disk and have a distance of $2 R_{\mathrm{o}}$, which is illustrated in Fig. 8.

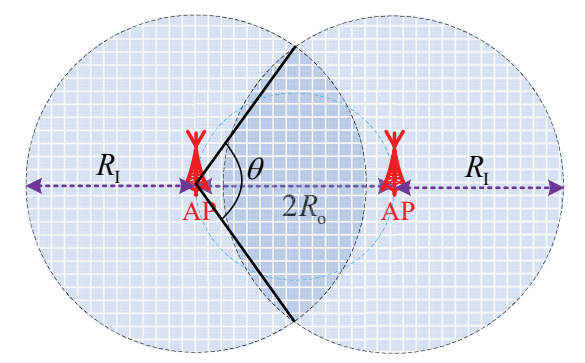

Fig. 8. Illustration of two-AP deployment for calculating $\bar{\xi}_{2}$.

From Fig. 8, we have

$$
\bar{S}_{2}=2 \pi R_{\mathrm{I}}^{2}-\bar{S}_{2 \mathrm{Lap}},
$$


where $\bar{S}_{2 \mathrm{Lap}}$ is the overlapping area of the two APs' receiving coverage disks with a radius of $R_{\mathrm{I}}$, which contains two identical circular segment areas with a radius of $R_{\mathrm{I}}$ and angle of $\theta$.

With some straightforward calculations, we have $\theta=2 \arccos \frac{R_{\mathrm{o}}}{R_{\mathrm{I}}}$ and $\bar{S}_{2 \mathrm{Lap}}=\theta R_{\mathrm{I}}^{2}-R_{\mathrm{I}}^{2} \sin \theta$. As a result, it is obtained that

$$
\bar{S}_{2}=2 \pi R_{\mathrm{I}}^{2}-\left(\theta R_{\mathrm{I}}^{2}-R_{\mathrm{I}}^{2} \sin \theta\right)
$$

Substituting (25) into (14), (18) can be obtained.

B. calculating $\bar{\xi}_{3}$

$\bar{\xi}_{3}$ is achieved when $p=3$ serving APs located in the disk with a radius of $R_{\mathrm{o}}$ have the largest total receiving coverage $\bar{S}_{3}$. This happens when the three APs are located at the edge of the disk and form an equilateral triangle where all the three sides are equal to $\sqrt{3} R_{\mathrm{o}}$, which is illustrated in Fig. 9.

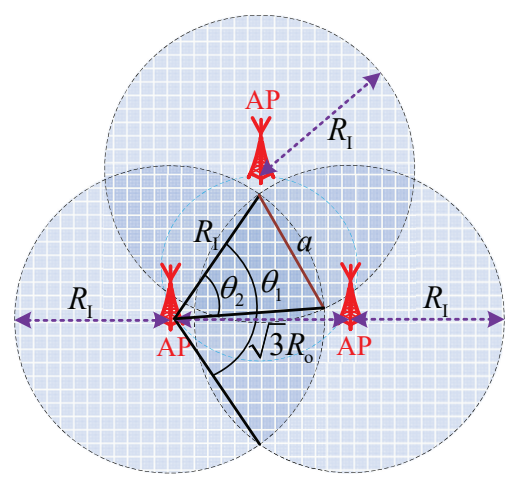

Fig. 9. Illustration of three-AP deployment for calculating $\bar{\xi}_{3}$.

Evidently, we have

$$
\bar{S}_{3}=3 \pi R_{\mathrm{I}}^{2}-3 \bar{S}_{2 \mathrm{Lap}}^{\prime}+\bar{S}_{3 \mathrm{Lap}}
$$

where $\bar{S}_{2 \mathrm{Lap}}^{\prime}$ is the overlapping area of any two APs' receiving coverage disks, which contains two identical circular segment areas with a radius of $R_{\mathrm{I}}$ and angle of $\theta_{1}$, and $\bar{S}_{3 \mathrm{Lap}}$ is the overlapping area of the three APs' receiving coverage disks, which contains three identical circular segment areas with a radius of $R_{\mathrm{I}}$ and angle of $\theta_{2}$ and one equilateral triangle area with each side of $a$. 
From Fig. 9, with some tedious and straightforward calculations, we obtain that $\theta_{1}=2 \arccos \frac{\sqrt{3} R_{\mathrm{o}}}{2 R_{\mathrm{I}}}$, $a=\sqrt{3}\left|\sqrt{R_{\mathrm{I}}^{2}-\frac{3 R_{\mathrm{o}}^{2}}{4}}-\frac{R_{\mathrm{o}}}{2}\right|$, and $\theta_{2}=2 \arcsin \frac{a}{2 R_{\mathrm{I}}}$. Thus, $\bar{S}_{2 \mathrm{Lap}}^{\prime}$ and $\bar{S}_{3 \text { Lap }}$ can be calculated as

$$
\bar{S}_{2 \mathrm{Lap}}^{\prime}=\theta_{1} R_{\mathrm{I}}^{2}-R_{\mathrm{I}}^{2} \sin \theta_{1}
$$

and

$$
\bar{S}_{3 \text { Lap }}=\frac{3}{2} \theta_{2} R_{\mathrm{I}}^{2}-\frac{3 a}{2} \sqrt{R_{\mathrm{I}}^{2}-\frac{a^{2}}{4}}+\frac{\sqrt{3} a^{2}}{4}
$$

respectively. With (26), (27), and (28), (19) can be obtained.

\section{REFERENCES}

[1] J. Ding, M. Nemati, C. Ranaweera, and J. Choi, "IoT connectivity technologies and applications: A survey," IEEE Access, vol. 8, pp. 67646-67673, 2020.

[2] W. Jiang, B. Han, M. A. Habibi, and H. D. Schotten, "The road towards 6G: A comprehensive survey," IEEE Open Journal of the Communications Society, vol. 2, pp. 334-366, 2021.

[3] B. A. Homssi, A. Al-Hourani, Z. Krusevac, and W. S. T. Rowe, "Machine learning framework for sensing and modeling interference in IoT frequency bands," IEEE Internet of Things Journal, vol. 8, no. 6, pp. 4461-4471, 2021.

[4] 3rd Generation Partnership Project (3GPP), Evolved Universal Terrestrial Radio Access (EUTRA) and Evolved Universal Terrestrial Radio Access Network (EUTRAN); Overall Description, TS 36.300 v.16.5.0, March 2021.

[5] 5G Americas, "The 5G evolution: 3GPP releases 16-17," 5G Americas, 2020. https://www.5gamericas.org/5g-evolution3gpp-releases-16-17.

[6] J. Choi, J. Ding, N. P. Le, and Z. Ding, "Grant-free random access in machine-type communication: approaches and challenges," IEEE Wireless Communications, pp. 1-6, 2021 (accepted).

[7] J. Kim, G. Lee, S. Kim, T. Taleb, S. Choi, and S. Bahk, "Two-step random access for 5G system: Latest trends and challenges," IEEE Network, vol. 35, no. 1, pp. 273-279, 2021.

[8] T. L. Marzetta, "Noncooperative cellular wireless with unlimited numbers of base station antennas," IEEE Transactions on Wireless Communications, vol. 9, pp. 3590-3600, November 2010.

[9] L. Lu, G. Y. Li, A. L. Swindlehurst, A. Ashikhmin, and R. Zhang, "An overview of massive MIMO: Benefits and challenges," IEEE J. Selected Topics in Signal Processing, vol. 8, no. 5, pp. 742-758, 2014.

[10] T. Marzetta, Fundamentals of Massive MIMO. Fundamentals of Massive MIMO, Cambridge University Press, 2016.

[11] Q. Zhang, S. Jin, and H. Zhu, "A hybrid-grant random access scheme in massive MIMO systems for IoT," IEEE Access, vol. 8, pp. 88487-88497, 2020.

[12] H. Jiang, D. Qu, J. Ding, and T. Jiang, "Multiple preambles for high success rate of grant-free random access with massive MIMO,” IEEE Trans. Wireless Communications, vol. 18, pp. 4779-4789, Oct 2019.

[13] J. Choi, "An approach to preamble collision reduction in grant-free random access with massive MIMO," IEEE Trans. Wireless Communications, vol. 20, no. 3, pp. 1557-1566, 2021.

[14] J. Ding and J. Choi, "Triangular non-orthogonal random access in mMIMO systems," IEEE Transactions on Communications, vol. 68, no. 11, pp. 6918-6931, 2020.

[15] J. H. Sørensen, E. de Carvalho, C. Stefanovic, and P. Popovski, "Coded pilot random access for massive MIMO systems," IEEE Trans. Wireless Communications, vol. 17, no. 12, pp. 8035-8046, 2018. 
[16] X.-H. You, D.-M. Wang, B. Sheng, X.-Q. Gao, X.-S. Zhao, and M. Chen, "Cooperative distributed antenna systems for mobile communications [coordinated and distributed MIMO]," IEEE Wireless Communications, vol. 17, no. 3, pp. 35-43, 2010.

[17] U. Madhow, D. R. Brown, S. Dasgupta, and R. Mudumbai, "Distributed massive MIMO: Algorithms, architectures and concept systems," in 2014 Information Theory and Applications Workshop (ITA), pp. 1-7, 2014.

[18] H. Q. Ngo, A. Ashikhmin, H. Yang, E. G. Larsson, and T. L. Marzetta, "Cell-free massive mimo versus small cells," IEEE Transactions on Wireless Communications, vol. 16, no. 3, pp. 1834-1850, 2017.

[19] G. Interdonato, G. Björnson, H. Q. Ngo, P. Frenger, and E. G. Larsson, "Ubiquitous cell-free Massive MIMO communications," EURASIP Journal on Wireless Communications and Networking, vol. 197, pp. 1-13, 2019.

[20] J. Zhang, S. Chen, Y. Lin, J. Zheng, B. Ai, and L. Hanzo, "Cell-free massive MIMO: A new next-generation paradigm," IEEE Access, vol. 7, pp. 99878-99888, 2019.

[21] I. F. Akyildiz, A. Kak, and S. Nie, "6G and beyond: The future of wireless communications systems," IEEE Access, vol. 8, pp. 133995-134030, 2020.

[22] J. Choi, "Compressive random access for MTC in distributed input distributed output systems," in 2017 IEEE 85th Vehicular Technology Conference (VTC Spring), pp. 1-5, June 2017.

[23] Z. Chen and E. Björnson, "Channel hardening and favorable propagation in cell-free massive MIMO with stochastic geometry," IEEE Trans. Communications, vol. 66, pp. 5205-5219, Nov 2018.

[24] J. Ding, D. Qu, P. Liu, and J. Choi, "Machine learning enabled preamble collision resolution in distributed massive MIMO," IEEE Transactions on Communications, vol. 69, no. 4, pp. 2317-2330, 2021.

[25] W. Zeng, Y. He, B. Li, and S. Wang, "Pilot assignment for cell free massive MIMO systems using a weighted graphic framework," IEEE Transactions on Vehicular Technology, vol. 70, no. 6, pp. 6190-6194, 2021.

[26] J. Ding, D. Kong, and D. Qu, "Improved tabu-search preamble assignment in cell-free massive MIMO systems," in 2021 International Wireless Communications and Mobile Computing (IWCMC), pp. 718-723, 2021.

[27] S. Buzzi, C. D’Andrea, M. Fresia, Y.-P. Zhang, and S. Feng, "Pilot assignment in cell-free massive MIMO based on the Hungarian algorithm," IEEE Wireless Communications Letters, vol. 10, no. 1, pp. 34-37, 2021.

[28] A. Papazafeiropoulos, P. Kourtessis, M. D. Renzo, S. Chatzinotas, and J. M. Senior, "Performance analysis of cell-free massive MIMO systems: A stochastic geometry approach,” IEEE Transactions on Vehicular Technology, vol. 69, no. 4, pp. 3523-3537, 2020.

[29] S. Buzzi and C. D’Andrea, "Cell-free massive MIMO: User-centric approach," IEEE Wireless Communications Letters, vol. 6, no. 6, pp. 706-709, 2017.

[30] S. N. Chiu, D. Stoyan, W. S. Kendall, and J. Mecke, Stochastic geometry and its applications. John Wiley \& Sons, 2013.

[31] 3GPP TR 36.814, Evolved Universal Terrestrial Radio Access (E-UTRA); Further advancements for E-UTRA physical layer aspects (Release 9), 2017.

[32] J. Choi, "Multi-channel estimation of devices with preamble collision in distributed MTC," in 2021 IEEE Statistical Signal Processing Workshop (SSP), pp. 511-515, 2021.

[33] A. Al-Hourani, S. Kandeepan, and S. Lardner, "Optimal LAP altitude for maximum coverage," IEEE Wireless Communications Letters, vol. 3, no. 6, pp. 569-572, 2014.

[34] J. F. C. Kingman, Poisson Processes. Wiley Online Library, 1993.

[35] J. Ding, D. Qu, H. Jiang, and T. Jiang, "Success probability of grant-free random access with massive MIMO," IEEE Internet of Things J., vol. 6, pp. 506-516, Feb 2019.

[36] Y. Liu, Y. Deng, M. Elkashlan, A. Nallanathan, and G. K. Karagiannidis, "Analyzing grant-free access for URLLC service," IEEE Journal on Selected Areas in Communications, vol. 39, no. 3, pp. 741-755, 2021. 